\title{
Density functional theory study of vibrational spectra, and assignment of fundamental modes of dacarbazine
}

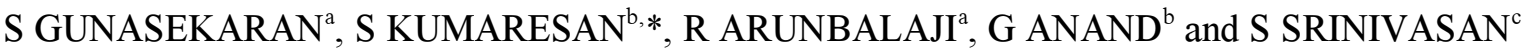 \\ ${ }^{a}$ Spectrophysics Research Laboratory, Pachaiyappa's College, Chennai 600030 \\ ${ }^{\mathrm{b}}$ Department of Physics, Arulmigu Meenakshi Amman College of Engineering, Vadamavandal 604410 \\ ${ }^{\mathrm{c}}$ Department of Physics, Arupadai Vedu Institute of Engineering Technology, Paiyanoor 603104 \\ e-mail: yeskay72@gmail.com
}

MS received 6 November 2007; revised 9 February 2008

\begin{abstract}
The FTIR and FT Raman spectra of dacarbazine were recorded in the regions 4000-400 and $3500-100 \mathrm{~cm}^{-1}$, respectively. The optimized geometry, wavenumber, polarizability and several thermodynamic properties of dacarbazine were studied using ab initio Hartree-Fock, MP2 and DFT methods. A complete vibrational assignment aided by the theoretical harmonic wavenumber analysis was proposed. The calculated harmonic vibrational frequencies were compared with experimental FTIR and FT Raman spectra. Based on the comparison between calculated and experimental results and the comparison with related molecules, assignments of fundamental vibrational modes were made. The X-ray geometry and experimental frequencies were compared with the results of theoretical calculations.
\end{abstract}

Keywords. FTIR; FT-Raman; density functional theory; dacarbazine.

\section{Introduction}

Dacarbazine, used as antineoplastic in the treatment of tumors. It belongs to the group of medicines called alkylating agents. It is used in the treatment of cancer of the lymph system and malignant melanoma a type of skin cancer. Dacarbazine, (DTIC-NSC-45388) is the single most active agent for the treatment of malignant melanoma. In addition to dacarbazine, other dimethyltriazenes have shown to demonstrate antitumor properties. ${ }^{1-5}$ Literature survey reveals that to the best of our knowledge, no $a b$ initio HF/MP2/ DFT frequency calculations of dacarbazine have been reported so far.

It is difficult to interpret the spectra of this molecule because of their complexity and low symmetry. In the interest of these physiological applications and the consequent in their qualitative and quantitative characterization in aqueous solution, the quantum mechanical calculations of this compound were thoroughly investigated. In this study, density functional theory (DFT) by using B3LYP hybrid functional, Moller-Plesset (MP2) and ab initio HartreeFock (HF) computations of the vibrational spectrum, the molecular geometry, the atomic charges and mole-

\footnotetext{
*For correspondence
}

cular polarizability calculations were carried out for dacarbazine molecule. The experimental geometric data of the molecule were taken from the Cambridge crystallographic database. ${ }^{6}$ Ab initio HF and density functional theory (DFT) calculations were performed to support our wavenumber assignment. Density functional calculations are reported to provide excellent vibrational wavenumber of organic compounds if the calculated frequencies are scaled to compensate for the approximate treatment of electron correlation, for basis set deficiencies and for the anharmonicity. $^{7-10}$

\section{Experimental}

The compound dacarbazine was purchased from Messrs Sigma-Aldrich Chemical Company, USA with more than $98 \%$ purity and was used as such without further purification to record FTIR and FT Raman spectra. The FTIR spectrum of the compound was recorded in the region $4000-400 \mathrm{~cm}^{-1}$ in evacuation mode on Bruker IFS 66V spectrophotometer using $\mathrm{KBr}$ pellet technique (solid phase) with $4.0 \mathrm{~cm}^{-1}$ resolution. The FT Raman spectrum was recorded using $1064 \mathrm{~nm}$ line of Nd:YAG laser as excitation wavelength in the region $3500-100 \mathrm{~cm}^{-1}$ on Bruker IFS 66V spectrometer equipped with FRA 


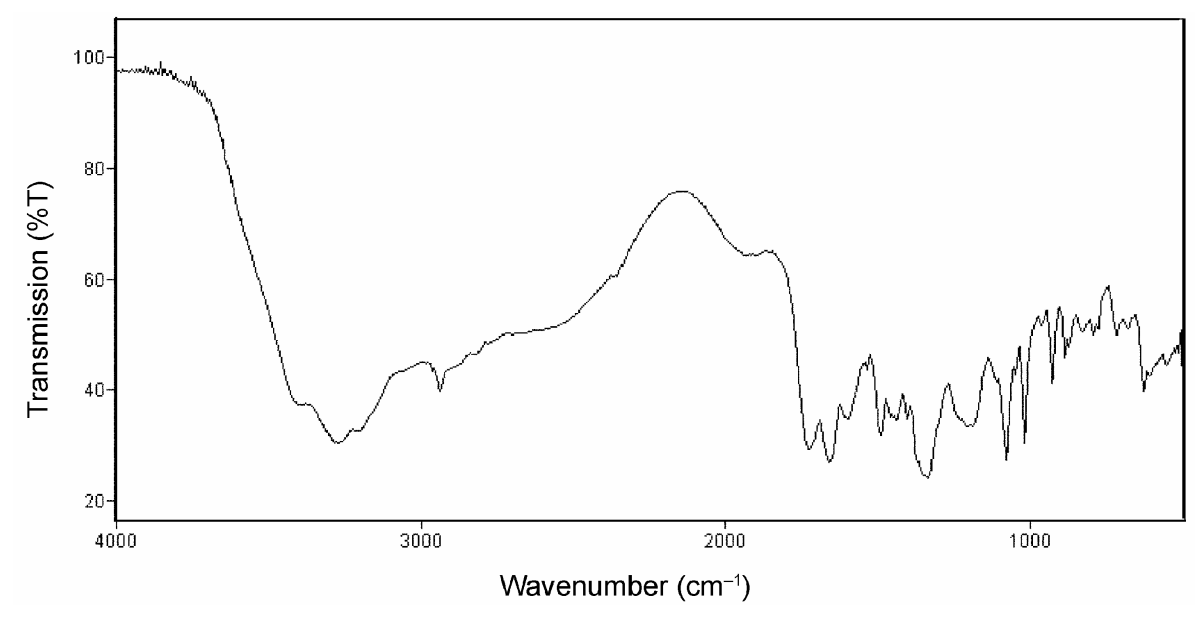

Figure 1. FTIR spectrum of dacarbazine.

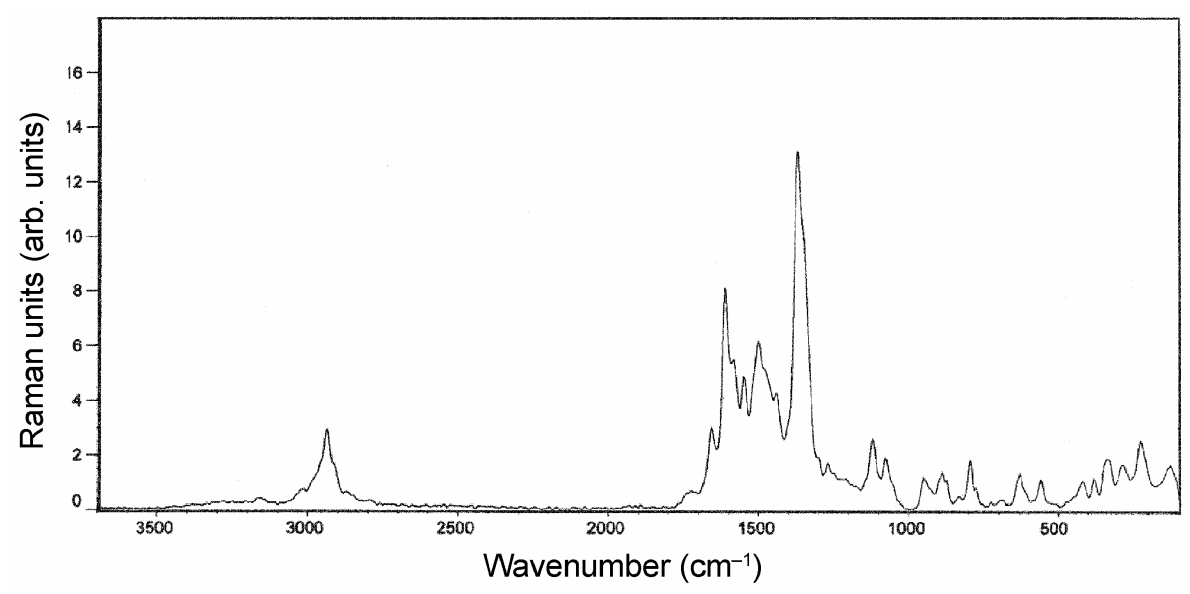

Figure 2. FT-Raman spectrum of dacarbazine.

106 FT Raman module accessory. The experimental FTIR and FT Raman spectra of dacarbazine are presented in figures 1 and 2 .

\section{Computational methods}

The entire calculations performed at HF, MP2 and B3LYP levels on an AMD 4000+/3.2 GHz personal computer using Gaussian $03 \mathrm{~W}$ program package. ${ }^{11}$ Initial geometry generated from standard geometrical parameters minimized without any constraint in the potential energy surface at HF level, adopting the standard 6-31G basis set. The optimized structural parameters used in the vibrational wavenumber calculations at the HF and DFT levels to characterize all stationary points as minima. Then, vibrationally averaged nuclear positions of dacarbazine used for harmonic vibrational wavenumber calculations resulting in IR and Raman wavenumbers together with in- tensities. Vibrational wavenumbers computed at DFT level have been adjudicated to be more reliable than those obtained by the computationally demanding Moller-Plesset perturbation methods. Finally, the calculated normal mode vibrational wavenumbers provide thermodynamic properties also through the principle of statistical mechanics. The assignments of the calculated wavenumbers supported by the animation option of chemcraft, a graphical interface for Gaussian programs, which gives a visual presentation of the shape of the vibrational modes. ${ }^{12}$

\section{Results and discussion}

\subsection{Geometric parameters}

In this work, we performed full geometry optimization of the title compound. The optimized structure parameters of the title compound calculated by $a b$ 
Table 1. Selected bond lengths $(\AA)$ and inter-axial angles $\left(^{\circ}\right)$ for dacarbazine.

\begin{tabular}{|c|c|c|c|c|c|c|}
\hline Molecular parameter & $X-r a y^{a}$ & AM1 & $\mathrm{HF} / 6-31 \mathrm{G}$ & B3LYP/6-31G & B3LYP/6-311G & $\mathrm{MP} 2 / 6-31 \mathrm{G}$ \\
\hline \multicolumn{7}{|l|}{ Bond length } \\
\hline $\mathrm{C}_{1}-\mathrm{N}_{2}$ & $1 \cdot 330$ & $1 \cdot 349$ & $1 \cdot 297$ & $1 \cdot 326$ & $1 \cdot 325$ & $1 \cdot 260$ \\
\hline $\mathrm{C}_{1}-\mathrm{N}_{5}$ & $1 \cdot 380$ & $1 \cdot 396$ & $1 \cdot 365$ & $1 \cdot 378$ & $1 \cdot 376$ & $1 \cdot 266$ \\
\hline $\mathrm{C}_{1}-\mathrm{H}_{14}$ & $1 \cdot 080$ & $1 \cdot 095$ & $1 \cdot 064$ & $1 \cdot 077$ & $1 \cdot 074$ & $1 \cdot 100$ \\
\hline $\mathrm{C}_{3}-\mathrm{C}_{4}$ & $1 \cdot 390$ & $1 \cdot 428$ & $1 \cdot 365$ & $1 \cdot 392$ & $1 \cdot 389$ & $1 \cdot 346$ \\
\hline $\mathrm{C}_{3}-\mathrm{C}_{6}$ & $1 \cdot 470$ & $1 \cdot 480$ & $1 \cdot 467$ & $1 \cdot 473$ & $1 \cdot 472$ & $1 \cdot 351$ \\
\hline $\mathrm{N}_{5}-\mathrm{H}_{15}$ & $0 \cdot 990$ & $0 \cdot 986$ & 0.990 & $1 \cdot 008$ & $1 \cdot 005$ & $1 \cdot 009$ \\
\hline $\mathrm{C}_{6}-\mathrm{O}_{7}$ & $1 \cdot 230$ & $1 \cdot 247$ & $1 \cdot 231$ & $1 \cdot 254$ & $1 \cdot 254$ & $1 \cdot 208$ \\
\hline $\mathrm{C}_{6}-\mathrm{N}_{8}$ & $1 \cdot 380$ & $1 \cdot 376$ & $1 \cdot 351$ & $1 \cdot 369$ & $1 \cdot 367$ & $1 \cdot 369$ \\
\hline $\mathrm{N}_{8}-\mathrm{H}_{16}$ & $0 \cdot 990$ & $0 \cdot 988$ & $0 \cdot 991$ & $1 \cdot 009$ & $1 \cdot 006$ & $1 \cdot 012$ \\
\hline $\mathrm{N}_{8}-\mathrm{H}_{17}$ & $0 \cdot 990$ & $0 \cdot 989$ & 0.991 & 1.007 & 1.004 & $1 \cdot 012$ \\
\hline $\mathrm{N}_{9}-\mathrm{N}_{10}$ & $1 \cdot 250$ & $1 \cdot 250$ & $1 \cdot 248$ & $1 \cdot 298$ & $1 \cdot 299$ & $1 \cdot 248$ \\
\hline $\mathrm{N}_{10}-\mathrm{N}_{11}$ & $1 \cdot 340$ & $1 \cdot 335$ & $1 \cdot 310$ & $1 \cdot 338$ & $1 \cdot 339$ & $1 \cdot 352$ \\
\hline $\mathrm{N}_{11}-\mathrm{C}_{12}$ & $1 \cdot 470$ & $1 \cdot 460$ & 1.455 & 1.465 & $1 \cdot 464$ & 1.438 \\
\hline $\mathrm{N}_{11}-\mathrm{C}_{13}$ & $1 \cdot 460$ & 1.460 & $1 \cdot 448$ & 1.456 & 1.456 & 1.438 \\
\hline $\mathrm{C}_{12}-\mathrm{H}_{18}$ & $1 \cdot 100$ & $1 \cdot 123$ & $1 \cdot 081$ & 1.095 & $1 \cdot 091$ & $1 \cdot 113$ \\
\hline $\mathrm{C}_{13}-\mathrm{H}_{22}$ & $1 \cdot 100$ & $1 \cdot 121$ & $1 \cdot 078$ & 1.090 & $1 \cdot 086$ & $1 \cdot 113$ \\
\hline $\mathrm{C}_{13}-\mathrm{H}_{23}$ & $1 \cdot 100$ & $1 \cdot 122$ & 1.083 & 1.097 & 1.092 & $1 \cdot 113$ \\
\hline $\mathrm{CC}$ & & 0.9944 & 0.9978 & 0.9994 & 0.9998 & 0.9965 \\
\hline MAD & & $0 \cdot 0169$ & $0 \cdot 0197$ & $0 \cdot 0033$ & $0 \cdot 0197$ & $0 \cdot 1852$ \\
\hline RMS & & $0 \cdot 0204$ & $0 \cdot 0224$ & 0.0045 & 0.0024 & $0 \cdot 0036$ \\
\hline \multicolumn{7}{|l|}{ Bond angle } \\
\hline $\mathrm{N}_{2}-\mathrm{C}_{1}-\mathrm{N}_{5}$ & $110 \cdot 450$ & 111.935 & $110 \cdot 594$ & $110 \cdot 874$ & $110 \cdot 844$ & $111 \cdot 004$ \\
\hline $\mathrm{N}_{2}-\mathrm{C}_{1}-\mathrm{H}_{14}$ & $125 \cdot 720$ & $125 \cdot 372$ & $126 \cdot 171$ & $125 \cdot 874$ & $125 \cdot 764$ & $124 \cdot 496$ \\
\hline $\mathrm{C}_{1}-\mathrm{N}_{2}-\mathrm{C}_{3}$ & $107 \cdot 080$ & $106 \cdot 138$ & $107 \cdot 005$ & $106 \cdot 479$ & $106 \cdot 453$ & 103.997 \\
\hline $\mathrm{N}_{5}-\mathrm{C}_{1}-\mathrm{H}_{14}$ & $123 \cdot 820$ & $122 \cdot 693$ & $123 \cdot 236$ & $123 \cdot 252$ & $123 \cdot 392$ & $124 \cdot 500$ \\
\hline $\mathrm{N}_{2}-\mathrm{C}_{3}-\mathrm{C}_{6}$ & $121 \cdot 390$ & $124 \cdot 281$ & $120 \cdot 437$ & $120 \cdot 372$ & $120 \cdot 14$ & $123 \cdot 593$ \\
\hline $\mathrm{C}_{3}-\mathrm{C}_{4}-\mathrm{N}_{9}$ & $127 \cdot 770$ & $125 \cdot 339$ & $129 \cdot 919$ & $129 \cdot 782$ & $129 \cdot 787$ & $129 \cdot 401$ \\
\hline $\mathrm{C}_{3}-\mathrm{C}_{6}-\mathrm{O}_{7}$ & $123 \cdot 570$ & $122 \cdot 386$ & $123 \cdot 365$ & $123 \cdot 856$ & $123 \cdot 927$ & 119.997 \\
\hline $\mathrm{N}_{5}-\mathrm{C}_{4}-\mathrm{N}_{9}$ & $126 \cdot 650$ & $129 \cdot 202$ & $124 \cdot 378$ & 124.899 & $124 \cdot 865$ & $129 \cdot 409$ \\
\hline $\mathrm{C}_{4}-\mathrm{N}_{5}-\mathrm{H}_{15}$ & $125 \cdot 460$ & $126 \cdot 296$ & $124 \cdot 607$ & $123 \cdot 420$ & $123 \cdot 384$ & $120 \cdot 001$ \\
\hline $\mathrm{C}_{4}-\mathrm{N}_{9}-\mathrm{N}_{10}$ & $116 \cdot 820$ & $119 \cdot 263$ & $115 \cdot 196$ & $113 \cdot 615$ & $113 \cdot 736$ & $120 \cdot 005$ \\
\hline $\mathrm{O}_{7}-\mathrm{C}_{6}-\mathrm{N}_{8}$ & $120 \cdot 700$ & $120 \cdot 185$ & $122 \cdot 341$ & $122 \cdot 897$ & $122 \cdot 706$ & $120 \cdot 000$ \\
\hline $\mathrm{C}_{6}-\mathrm{N}_{8}-\mathrm{H}_{16}$ & $118 \cdot 400$ & $121 \cdot 056$ & $120 \cdot 903$ & $119 \cdot 819$ & $119 \cdot 857$ & $119 \cdot 996$ \\
\hline $\mathrm{C}_{6}-\mathrm{N}_{8}-\mathrm{H}_{17}$ & $117 \cdot 280$ & $118 \cdot 547$ & $118 \cdot 551$ & $118 \cdot 832$ & $118 \cdot 764$ & $120 \cdot 003$ \\
\hline $\mathrm{N}_{9}-\mathrm{N}_{10}-\mathrm{N}_{11}$ & $116 \cdot 580$ & $119 \cdot 425$ & $116 \cdot 285$ & $113 \cdot 740$ & $113 \cdot 63$ & $109 \cdot 502$ \\
\hline $\mathrm{N}_{10}-\mathrm{N}_{11}-\mathrm{C}_{12}$ & $122 \cdot 300$ & $123 \cdot 978$ & $120 \cdot 858$ & $121 \cdot 131$ & $120 \cdot 988$ & $109 \cdot 446$ \\
\hline $\mathrm{N}_{10}-\mathrm{N}_{11}-\mathrm{C}_{13}$ & $115 \cdot 010$ & $115 \cdot 522$ & $117 \cdot 302$ & $116 \cdot 858$ & $116 \cdot 889$ & $109 \cdot 440$ \\
\hline $\mathrm{C}_{12}-\mathrm{N}_{11}-\mathrm{C}_{13}$ & $117 \cdot 780$ & $113 \cdot 380$ & $121 \cdot 840$ & $122 \cdot 010$ & $122 \cdot 123$ & $109 \cdot 506$ \\
\hline $\mathrm{N}_{11}-\mathrm{C}_{12}-\mathrm{H}_{18}$ & $111 \cdot 150$ & $111 \cdot 764$ & $110 \cdot 759$ & $110 \cdot 411$ & $110 \cdot 442$ & $109 \cdot 439$ \\
\hline $\mathrm{N}_{11}-\mathrm{C}_{12}-\mathrm{H}_{19}$ & $108 \cdot 370$ & $108 \cdot 144$ & $107 \cdot 953$ & $108 \cdot 283$ & $108 \cdot 295$ & $109 \cdot 457$ \\
\hline $\mathrm{N}_{11}-\mathrm{C}_{13}-\mathrm{H}_{22}$ & $110 \cdot 200$ & $111 \cdot 542$ & $108 \cdot 582$ & $107 \cdot 838$ & $107 \cdot 839$ & $109 \cdot 465$ \\
\hline $\mathrm{N}_{11}-\mathrm{C}_{13}-\mathrm{H}_{23}$ & $110 \cdot 150$ & $107 \cdot 023$ & $110 \cdot 822$ & $111 \cdot 002$ & 111.031 & $109 \cdot 447$ \\
\hline $\mathrm{H}_{18}-\mathrm{C}_{12}-\mathrm{H}_{19}$ & $109 \cdot 090$ & $109 \cdot 246$ & 109.937 & $110 \cdot 426$ & $110 \cdot 428$ & $109 \cdot 464$ \\
\hline $\mathrm{H}_{22}-\mathrm{C}_{13}-\mathrm{H}_{23}$ & $108 \cdot 560$ & $108 \cdot 733$ & 108.906 & $109 \cdot 124$ & $109 \cdot 067$ & $109 \cdot 202$ \\
\hline $\mathrm{CC}$ & & 0.9715 & 0.9814 & 0.9751 & 0.9746 & 0.9568 \\
\hline MAD & & $1 \cdot 3296$ & 1.0395 & $1 \cdot 3335$ & $1 \cdot 2750$ & $1 \cdot 3357$ \\
\hline RMS & & 1.7229 & $1 \cdot 4339$ & 1.7452 & 1.6729 & $1 \cdot 6150$ \\
\hline
\end{tabular}

${ }^{\mathrm{a}}$ Geometrical parameters determined with X-ray diffraction method from ref. [6]

$\mathrm{CC}$, Correlation coefficient; MAD, Mean arithmetic deviation; RMS, Root mean square error

initio, MP2, DFT and AM1 methods listed in table 1 are in accordance with atom numbering scheme given in figure $3 \mathrm{~b}$. The B3LYP method leads to ge- ometry parameters, which are close to experimental data. ${ }^{6}$ A statistical treatment of these data (see at the bottom of table 1) shows that for the bond lengths 

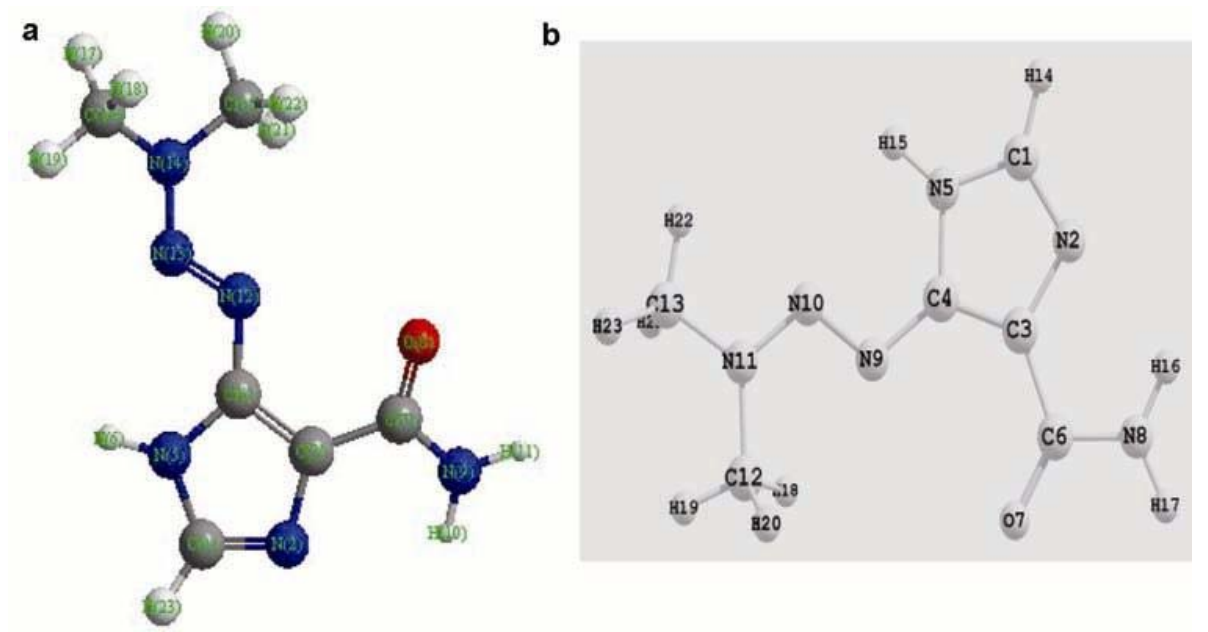

Figure 3. a. X-ray structure of dacarbazine. b. Geometry of the dacarbazine optimized at B3LYP/6-31G $(d, p)$.
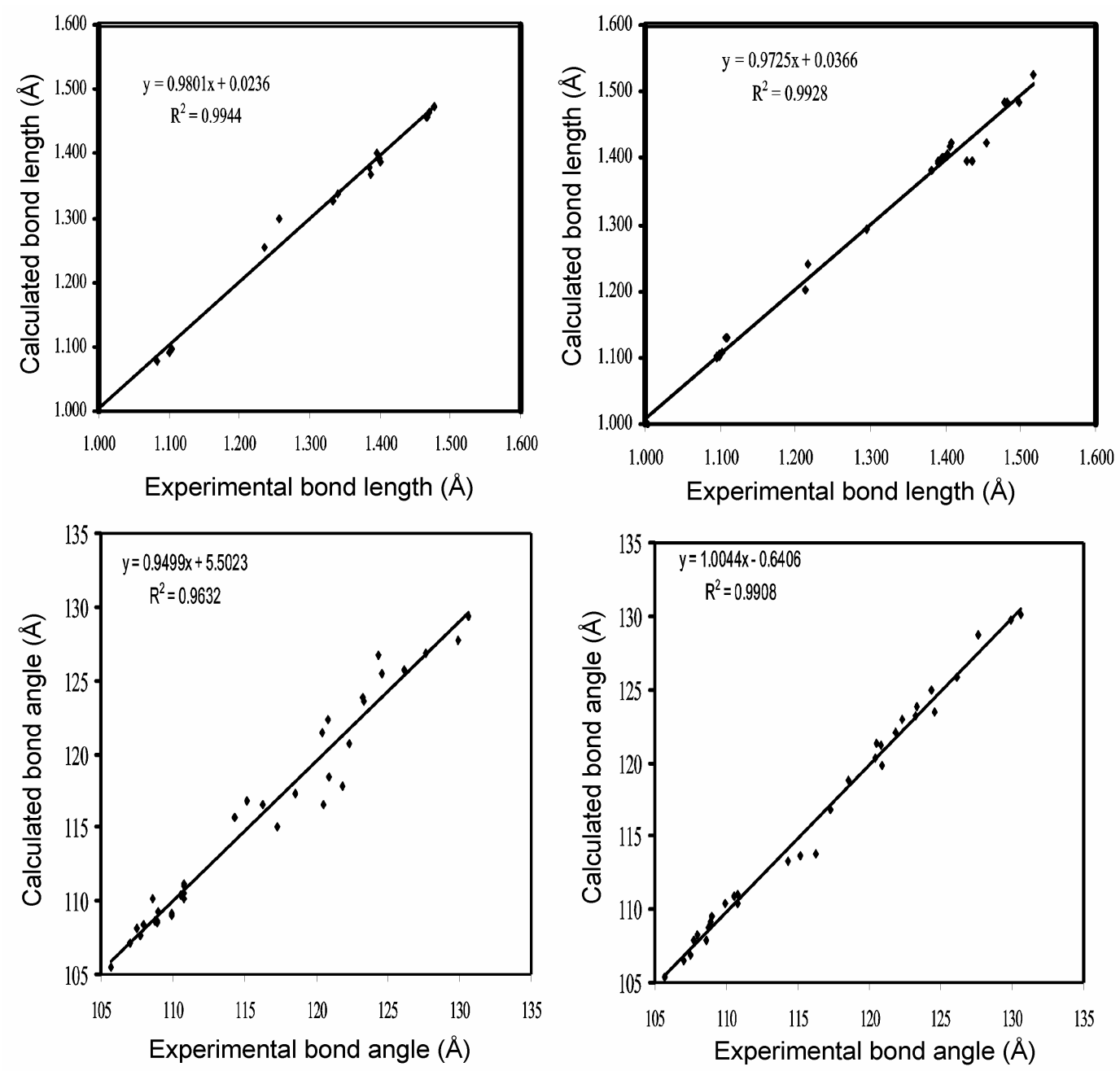

Figure 4. Calculated bond lengths and bond angles in comparison with experimental data.

$\mathrm{B} 3 \mathrm{LYP} / 6-31 \mathrm{G}(d, p)$ (figure 4) is slightly better than the $\mathrm{HF} / 6-31 \mathrm{G}(d, p)$ geometry. The correlation coefficient for bond lengths was 0.9994 for B3LYP/6-
$31 \mathrm{G}(d, p)$ method. The agreement for bond angles is not as good as that for the bond distances. The slight variation with the experimental value is due to the 
Table 2. Observed FTIR, FT-Raman and calculated wave numbers for dacarbazine using HF/6-31G(d, p), B3LYP/6$31 \mathrm{G}(d, p)$ and MP2/6-31G $(d, p)$ methods.

\begin{tabular}{|c|c|c|c|c|c|c|c|c|}
\hline \multirow[b]{3}{*}{ Vibrational assignment } & \multicolumn{8}{|c|}{ Calculated wave number $\left(\mathrm{cm}^{-1}\right)$} \\
\hline & \multicolumn{2}{|c|}{$\begin{array}{c}\text { Observed } \\
\text { wave number }\left(\mathrm{cm}^{-1}\right)\end{array}$} & \multicolumn{2}{|c|}{$\begin{array}{c}\mathrm{HF} \\
6-31 \mathrm{G}(d, p)\end{array}$} & \multicolumn{2}{|c|}{$\begin{array}{c}\text { B3LYP } \\
6-31 \mathrm{G}(d, p)\end{array}$} & \multicolumn{2}{|c|}{$\begin{array}{c}\text { MP2 } \\
6-31 \mathrm{G}(d, p)\end{array}$} \\
\hline & FTIR & $\begin{array}{l}\text { FT- } \\
\text { Raman }\end{array}$ & $\begin{array}{l}\text { Wave } \\
\text { number }\end{array}$ & $\begin{array}{c}\text { Rel } \\
\text { Intensity }\end{array}$ & $\begin{array}{l}\text { Wave } \\
\text { number }\end{array}$ & $\begin{array}{c}\text { Rel } \\
\text { Intensity }\end{array}$ & $\begin{array}{l}\text { Wave } \\
\text { number }\end{array}$ & $\begin{array}{c}\text { Rel } \\
\text { Intensity }\end{array}$ \\
\hline$\gamma_{\text {as }} \mathrm{NH}_{2}$ & $3370(w)$ & $3371(w)$ & 3596 & 13 & 3376 & 8 & 3389 & 0 \\
\hline$\gamma \mathrm{NH}$ & $3321(w)$ & & 3544 & 17 & 3314 & 9 & 3380 & 38 \\
\hline$\gamma_{\mathrm{s}} \mathrm{NH}_{2}$ & $3232(w)$ & & 3466 & 14 & 3238 & 8 & 3207 & 2 \\
\hline$\gamma_{\mathrm{s}} \mathrm{CH}$ & $2957(w)$ & $2937(\mathrm{~m})$ & 3140 & 0 & 2978 & 0 & 3132 & 0 \\
\hline$\gamma_{\mathrm{as}} \mathrm{CH}_{3}$ & $2869(w)$ & & 2994 & 2 & 2861 & 2 & 3129 & 0 \\
\hline$\gamma_{\mathrm{as}} \mathrm{CH}_{3}$ & $2853(w)$ & $2851(w)$ & 2988 & 3 & 2858 & 1 & 3124 & 5 \\
\hline$\gamma_{\mathrm{as}} \mathrm{CH}_{2}$ & $2819(w)$ & & 2962 & 2 & 2814 & 1 & 3098 & 1 \\
\hline$\gamma_{\mathrm{as}} \mathrm{CH}_{2}$ & & $2780(w)$ & 2930 & 6 & 2786 & 5 & 3092 & 2 \\
\hline$\gamma_{\mathrm{s}} \mathrm{CH}_{3}$ & $2755(w)$ & & 2897 & 6 & 2756 & 5 & 2947 & 1 \\
\hline$\gamma_{\mathrm{s}} \mathrm{CH}_{3}$ & & $2739(w)$ & 2877 & 13 & 2734 & 14 & 2943 & 4 \\
\hline$\gamma \mathrm{C}=\mathrm{O}$ & $1612(s)$ & $1613(s)$ & 1708 & 70 & 1620 & 80 & 1610 & 53 \\
\hline$\gamma \mathrm{C}=\mathrm{N}$ & $1510(w)$ & $1501(\mathrm{~m})$ & 1673 & 57 & 1516 & 53 & 1572 & 11 \\
\hline$\rho \mathrm{C}-\mathrm{NH}_{2}$ & $1496(s)$ & & 1657 & 11 & 1498 & 2 & 1509 & 1 \\
\hline$\rho \mathrm{NH}_{2}$ & $1448(w)$ & $1441(w)$ & 1567 & 67 & 1434 & 6 & 1495 & 8 \\
\hline$\gamma \operatorname{ringCN}$ & $1441(w)$ & & 1549 & 8 & 1420 & 9 & 1490 & 1 \\
\hline$\gamma \mathrm{CC}+\gamma$ ring $+\rho \mathrm{NH}_{2}$ & $1404(\mathrm{~m})$ & $1402(w)$ & 1543 & 0 & 1406 & 1 & 1483 & 2 \\
\hline$\gamma \mathrm{CC}+\alpha \mathrm{N}-\mathrm{CH}_{2}+\beta \mathrm{NCH}$ & $1394(w)$ & & 1531 & 6 & 1395 & 8 & 1450 & 13 \\
\hline$\delta_{\mathrm{as}} \mathrm{CH}_{3}+\gamma$ ring & $1383(w)$ & & 1517 & 1 & 1386 & 2 & 1416 & 5 \\
\hline$\delta_{\mathrm{as}} \mathrm{CH}_{3}+\gamma$ ring & & $1386(w)$ & 1503 & 2 & 1386 & 6 & 1398 & 2 \\
\hline$\delta_{\mathrm{as}} \mathrm{CH}_{3}+\gamma$ ring & & $1369(s)$ & 1499 & 1 & 1364 & 3 & 1383 & 11 \\
\hline$\delta_{\mathrm{s}} \mathrm{CH}_{3}+\gamma$ ring & $1337(m)$ & & 1483 & 1 & 1345 & 4 & 1373 & 11 \\
\hline$\delta_{\mathrm{s}} \mathrm{CH}_{3}+\gamma$ ring & $1295(w)$ & $1285(w)$ & 1456 & 16 & 1289 & 9 & 1245 & 48 \\
\hline$\delta_{\mathrm{s}} \mathrm{CH}_{3}+\gamma \operatorname{ring}+\beta \mathrm{NCH}$ & $1280(w)$ & & 1412 & 78 & 1282 & 100 & 1226 & 4 \\
\hline$\delta_{\mathrm{s}} \mathrm{CH}_{3}+\gamma$ ring & & $1263(w)$ & 1391 & 38 & 1266 & 1 & 1218 & 8 \\
\hline$\gamma \mathrm{CN}+\delta \mathrm{CH}$ & $1255(w)$ & & 1382 & 29 & 1255 & 1 & 1191 & 5 \\
\hline$\gamma$ ring $+\delta \mathrm{CH}$ & $1209(w)$ & & 1342 & 9 & 1223 & 2 & 1139 & 15 \\
\hline$\alpha \mathrm{NH}+\gamma \mathrm{NNN}$ & & $1166(w)$ & 1273 & 3 & 1166 & 1 & 1131 & 1 \\
\hline$\gamma \mathrm{NN}+\delta \mathrm{CH}_{3}$ & $1137(w)$ & $1121(w)$ & 1223 & 4 & 1130 & 4 & 1106 & 0 \\
\hline$\gamma \mathrm{NN}+\beta \mathrm{CH}$ & $1082(s)$ & $1078(w)$ & 1184 & 1 & 1087 & 0 & 1074 & 18 \\
\hline$\beta \mathrm{CH}+\delta \mathrm{CNC}$ & & $1064(w)$ & 1166 & 1 & 1067 & 0 & 1061 & 14 \\
\hline$\rho \mathrm{CH}_{2}+\beta \mathrm{CH}$ & $1052(w)$ & & 1145 & 0 & 1047 & 0 & 1040 & 2 \\
\hline$\rho \mathrm{C}-\mathrm{NH}_{2}+\delta \mathrm{CNC}$ & $1022(s)$ & $1028(w)$ & 1132 & 24 & 1027 & 8 & 996 & 1 \\
\hline$\rho \mathrm{CH}_{2}+\delta \mathrm{CNC}$ & $966(w)$ & & 1113 & 7 & 1026 & 8 & 987 & 1 \\
\hline$\rho \mathrm{CH}_{3}+\beta \mathrm{CH}$ & $930(s)$ & $950(w)$ & 1086 & 3 & 999 & 18 & 974 & 19 \\
\hline$\rho \mathrm{CH}_{3}+\gamma$ ring & $889(m)$ & $889(w)$ & 1085 & 42 & 995 & 11 & 954 & 26 \\
\hline$\Delta \operatorname{ring}+\gamma \operatorname{ring}(\mathrm{CN})$ & $874(w)$ & $876(w)$ & 977 & 4 & 891 & 3 & 841 & 1 \\
\hline$\gamma \mathrm{N}-\mathrm{CH}_{3}+\alpha \mathrm{NNN}$ & $830(w)$ & $844(w)$ & 917 & 0 & 841 & 2 & 811 & 8 \\
\hline$\beta \mathrm{NCH}+\beta \mathrm{NCC}$ & $794(w)$ & $797(m)$ & 915 & 3 & 774 & 1 & 767 & 100 \\
\hline$\beta \mathrm{CH}$ & $745(w)$ & $743(w)$ & 857 & 14 & 748 & 4 & 726 & 9 \\
\hline$\gamma \mathrm{NN}+\gamma \mathrm{N}-\mathrm{CH}_{3}+\alpha \mathrm{CNC}$ & $718(w)$ & & 788 & 0 & 735 & 0 & 698 & 18 \\
\hline$\beta \mathrm{NH}+\beta \mathrm{CCO}$ & $682(w)$ & $670(w)$ & 759 & 2 & 665 & 4 & 638 & 14 \\
\hline$\beta(\mathrm{CCN}+\mathrm{NH})$ & $630(m)$ & $631(m)$ & 718 & 65 & 643 & 22 & 598 & 6 \\
\hline$\alpha \mathrm{NNN}+\alpha \mathrm{CNC}$ & $625(w)$ & & 690 & 7 & 635 & 18 & 548 & 2 \\
\hline$\beta \mathrm{NCO}+\beta \mathrm{NH}+\beta \mathrm{CH}$ & $622(w)$ & $610(w)$ & 690 & 4 & 635 & 2 & 589 & 8 \\
\hline$\tau \mathrm{C}-\mathrm{NH}_{2}+\beta \mathrm{NH}_{2}$ & $593(w)$ & & 629 & 19 & 594 & 13 & 493 & 15 \\
\hline$\rho \mathrm{NH}_{2}+\alpha \mathrm{NNH}+\alpha \mathrm{CCO}$ & $554(w)$ & $560(m)$ & 605 & 8 & 557 & 6 & 474 & 2 \\
\hline Ring deformation & $525(w)$ & & 578 & 10 & 525 & 3 & 463 & 30 \\
\hline$\beta \mathrm{NH}+\alpha \mathrm{NCH}$ & $514(w)$ & $510(w)$ & 564 & 3 & 512 & 23 & 407 & 8 \\
\hline$\alpha \mathrm{C}-\mathrm{NH}_{2}$ & & $420(w)$ & 441 & 2 & 410 & 2 & 369 & 4 \\
\hline
\end{tabular}


Table 2. (contd ...)

\begin{tabular}{|c|c|c|c|c|c|c|c|}
\hline \multirow[b]{3}{*}{ Vibrational assignment } & & \multicolumn{6}{|c|}{ Calculated wave number $\left(\mathrm{cm}^{-1}\right)$} \\
\hline & $\begin{array}{c}\text { Observed } \\
\text { wave number }\left(\mathrm{cm}^{-1}\right)\end{array}$ & \multicolumn{2}{|c|}{$\begin{array}{c}\mathrm{HF} \\
6-31 \mathrm{G}(d, p)\end{array}$} & \multicolumn{2}{|c|}{$\begin{array}{c}\text { B3LYP } \\
6-31 \mathrm{G}(d, p)\end{array}$} & \multicolumn{2}{|c|}{$\begin{array}{c}\mathrm{MP} 2 \\
6-31 \mathrm{G}(d, p)\end{array}$} \\
\hline & $\begin{array}{c}\text { FT- } \\
\text { Raman }\end{array}$ & $\begin{array}{l}\text { Wave } \\
\text { number }\end{array}$ & $\begin{array}{c}\text { Rel } \\
\text { Intensity }\end{array}$ & $\begin{array}{l}\text { Wave } \\
\text { number }\end{array}$ & $\begin{array}{c}\text { Rel } \\
\text { Intensity }\end{array}$ & $\begin{array}{l}\text { Wave } \\
\text { number }\end{array}$ & $\begin{array}{c}\text { Rel } \\
\text { Intensity }\end{array}$ \\
\hline $\operatorname{ringCNH}+\rho \mathrm{NH}_{2}+\beta \mathrm{NCO}$ & $381(m)$ & 415 & 0 & 383 & 0 & 349 & 9 \\
\hline$\alpha \mathrm{NNH}+\alpha \mathrm{CCO}$ & & 413 & 2 & 369 & 0 & 325 & 8 \\
\hline$\omega \mathrm{NH}_{2}$ & $337(m)$ & 393 & 0 & 361 & 1 & 285 & 3 \\
\hline$\Omega \mathrm{CH}_{3} \alpha \mathrm{CNH}$ & $285(w)$ & 315 & 2 & 292 & 2 & 256 & 3 \\
\hline$\beta \mathrm{C}-\mathrm{NH}_{2}$ & $226(\mathrm{~m})$ & 272 & 0 & 253 & 0 & 243 & 2 \\
\hline$\beta \mathrm{CNH}+\beta \mathrm{CH}_{2}$ & & 225 & 1 & 204 & 1 & 206 & 2 \\
\hline$\alpha \mathrm{NCO}+\beta \mathrm{NH}_{2}$ & & 202 & 3 & 181 & 2 & 188 & 1 \\
\hline$\tau \mathrm{CH}_{2}+\tau \mathrm{CH}_{3}$ & & 188 & 1 & 174 & 1 & 159 & 2 \\
\hline$\tau \mathrm{CH}_{3}+\beta \mathrm{NNN}+\beta \mathrm{NCH}+\beta \mathrm{NH}_{2}$ & $126(w)$ & 146 & 0 & 142 & 0 & 155 & 2 \\
\hline Lattice vibrations & & 113 & 0 & 98 & 0 & 144 & 2 \\
\hline$\beta \mathrm{NCO}+\omega \mathrm{CH}_{3}$ & & 78 & 1 & 71 & 1 & 90 & 0 \\
\hline$\omega \mathrm{CH}_{3}$ & & 63 & 0 & 58 & 2 & 63 & 1 \\
\hline$\omega \mathrm{CH}_{3}$ & & 56 & 2 & 47 & 0 & 46 & 1 \\
\hline$\omega \mathrm{CH}_{3}$ & & 38 & 0 & 23 & 0 & 36 & 0 \\
\hline
\end{tabular}

fact that the optimization performed in an isolated condition, whereas the crystal environment affected the experimental X-ray structure.

\subsection{Vibrational assignments}

The FT-IR and FT-Raman spectra of the title compound were shown in figures $1-2$. None of the predicted vibrational spectra have any imaginary frequency, implying that the optimized geometry is located at the local lowest point on the potential energy surface. We know that $a b$ initio, MP2 and DFT potentials systematically overestimate the vibrational wave numbers. These discrepancies corrected either by computing anharmonic corrections explicitly or by introducing a scaled field ${ }^{13}$ or directly scaling the calculated wave numbers with the proper factor. ${ }^{14}$ Considering systematic errors with scaling factor of 0.9051 , 0.9500 , and 0.9679 , we calibrated the vibrational wave numbers calculated by HF, MP2, and B3LYP, respectively. After scaled with a scaling factor, the deviation from experiments is less than $10 \mathrm{~cm}^{-1}$ with a few exceptions. Theoretical and experimental results of the title compound are shown in table 2. The DFT method is superior to HF and MP2 methods in terms of realistic reproduction of both band intensity distribution and general spectral features.

4.2a $C-O$ vibration: The characteristics infrared absorption frequencies of carbonyl group in ketones are normally strong in intensity and found in the region 1685-1660 $\mathrm{cm}^{-1}$. The carbon-oxygen double bond formed by $p_{\pi}-p_{\pi}$ between carbon and oxygen. Because of the different electro negativities of carbon and oxygen atoms, the bonding electrons not equally distributed between the two atoms. The lone pair of electrons on oxygen also determines the nature of the carbonyl group. ${ }^{15}$ The $\mathrm{CO}$ stretching vibration in dacarbazine has a scaled computed frequency of $1620 \mathrm{~cm}^{-1}$. This is in agreement with strong experimental frequency in FT-IR spectrum at $1612 \mathrm{~cm}^{-1}$. The $\mathrm{C}-\mathrm{O}$ out-of-plane bending vibration mode with the theoretical frequency of $142 \mathrm{~cm}^{-1}$ agrees well with experimental FT-Raman value. The above conclusions are in agreement with literature value. ${ }^{16}$

$4.2 \mathrm{~b} \mathrm{C}-\mathrm{NH}_{2}$ vibrations: The molecule under investigation possesses only one $\mathrm{NH}_{2}$ group and hence one expects one symmetric and one asymmetric $\mathrm{N}-$ $\mathrm{H}$ stretching vibrations in $\mathrm{NH}_{2}$ group. In all the primary aromatic amines, the $\mathrm{N}-\mathrm{H}$ stretching frequency occurs in the region $3300-3500 \mathrm{~cm}^{-1} .{ }^{17}$ Hence, the weak bands in IR spectrum were located at 3370 and $3232 \mathrm{~cm}^{-1}$ assigned to $\mathrm{N}-\mathrm{H}$ asymmetric and symmetric stretching vibrations, respectively in $\mathrm{NH}_{2}$ group. These assignments agree well with the earlier reports. ${ }^{18}$ The scaled $-\mathrm{NH}_{2}$ asymmetric and symmetric stretching are in the range of $3376-3238 \mathrm{~cm}^{-1}$ in B3LYP/6-31G $(d, p)$. The computed $-\mathrm{NH}_{2}$ scissoring vibration at $1498 \mathrm{~cm}^{-1}$ in $\mathrm{B} 3 \mathrm{LYP} / 6-31 \mathrm{G}(d, p)$ is in agreement with the expected experimental value at 
Table 3. Mulliken atomic charges of dacarbazine for different levels and basis sets.

\begin{tabular}{|c|c|c|c|c|c|}
\hline \multirow[b]{2}{*}{ Atom with numbering } & \multicolumn{3}{|c|}{ B3LYP } & \multirow{2}{*}{$\begin{array}{l}\mathrm{HF} \\
6-31 \mathrm{G}(d, p)\end{array}$} & \multirow{2}{*}{$\begin{array}{c}\mathrm{MP} 2 \\
6-31 \mathrm{G}(d, p)\end{array}$} \\
\hline & $6-31 \mathrm{G}^{*}$ & $6-311 \mathrm{G}(d, p)$ & $6-31 \mathrm{G}(d, p)$ & & \\
\hline $\mathrm{C}_{1}$ & $0 \cdot 2301$ & 0.2322 & $0 \cdot 1718$ & $0 \cdot 2941$ & $0 \cdot 3488$ \\
\hline $\mathrm{N}_{2}$ & -0.4117 & -0.4096 & $-0 \cdot 2461$ & -0.4753 & -0.6033 \\
\hline $\mathrm{C}_{3}$ & $-0 \cdot 1242$ & $-0 \cdot 1221$ & $-0 \cdot 3088$ & -0.0208 & 0.0533 \\
\hline $\mathrm{C}_{4}$ & 0.4759 & 0.4780 & $0 \cdot 3510$ & 0.5513 & 0.6092 \\
\hline $\mathrm{N}_{5}$ & -0.7332 & -0.7311 & -0.6565 & -0.8619 & -0.6908 \\
\hline $\mathrm{C}_{6}$ & 0.5961 & 0.5982 & 0.6378 & 0.7439 & 0.7671 \\
\hline $\mathrm{O}_{7}$ & -0.4137 & -0.4116 & $-0 \cdot 3642$ & -0.5735 & -0.6408 \\
\hline $\mathrm{N}_{8}$ & -0.7863 & -0.7842 & $-0 \cdot 8015$ & -0.9632 & -0.7656 \\
\hline $\mathrm{N}_{9}$ & $-0 \cdot 2519$ & $-0 \cdot 2498$ & $-0 \cdot 0254$ & $-0 \cdot 1720$ & -0.4278 \\
\hline $\mathrm{N}_{10}$ & -0.0217 & -0.0196 & $-0 \cdot 1173$ & $-0 \cdot 1319$ & -0.0184 \\
\hline $\mathrm{N}_{11}$ & $-0 \cdot 3670$ & $-0 \cdot 3649$ & -0.2311 & -0.4069 & -0.3996 \\
\hline $\mathrm{C}_{12}$ & $-0 \cdot 3103$ & $-0 \cdot 3082$ & 0.3895 & $-0 \cdot 2812$ & -0.1317 \\
\hline $\mathrm{C}_{13}$ & $-0 \cdot 3240$ & $-0 \cdot 3219$ & $-0 \cdot 3481$ & $-0 \cdot 2484$ & $-0 \cdot 1223$ \\
\hline $\mathrm{H}_{14}$ & $0 \cdot 2037$ & 0.2058 & $0 \cdot 1968$ & 0.2524 & $0 \cdot 1776$ \\
\hline $\mathrm{H}_{15}$ & $0 \cdot 3548$ & 0.3569 & $0 \cdot 3686$ & 0.4468 & $0 \cdot 3440$ \\
\hline $\mathrm{H}_{16}$ & $0 \cdot 3446$ & $0 \cdot 3467$ & 0.3274 & $0 \cdot 3954$ & 0.3377 \\
\hline $\mathrm{H}_{17}$ & $0 \cdot 3267$ & $0 \cdot 3288$ & $0 \cdot 3240$ & $0 \cdot 3832$ & $0 \cdot 3128$ \\
\hline $\mathrm{H}_{18}$ & $0 \cdot 2254$ & $0 \cdot 2275$ & $0 \cdot 2132$ & $0 \cdot 2055$ & $0 \cdot 1429$ \\
\hline $\mathrm{H}_{19}$ & $0 \cdot 1710$ & $0 \cdot 1731$ & $0 \cdot 1647$ & $0 \cdot 1507$ & $0 \cdot 1821$ \\
\hline $\mathrm{H}_{20}$ & $0 \cdot 2254$ & $0 \cdot 2275$ & $0 \cdot 1944$ & $0 \cdot 1920$ & $0 \cdot 1220$ \\
\hline $\mathrm{H}_{21}$ & 0.1973 & $0 \cdot 1994$ & $0 \cdot 1981$ & $0 \cdot 1868$ & 0.1238 \\
\hline $\mathrm{H}_{22}$ & $0 \cdot 1958$ & $0 \cdot 1979$ & $0 \cdot 1756$ & $0 \cdot 1786$ & $0 \cdot 1431$ \\
\hline $\mathrm{H}_{23}$ & $0 \cdot 1973$ & $0 \cdot 1994$ & $0 \cdot 1651$ & $0 \cdot 1544$ & $0 \cdot 1359$ \\
\hline
\end{tabular}

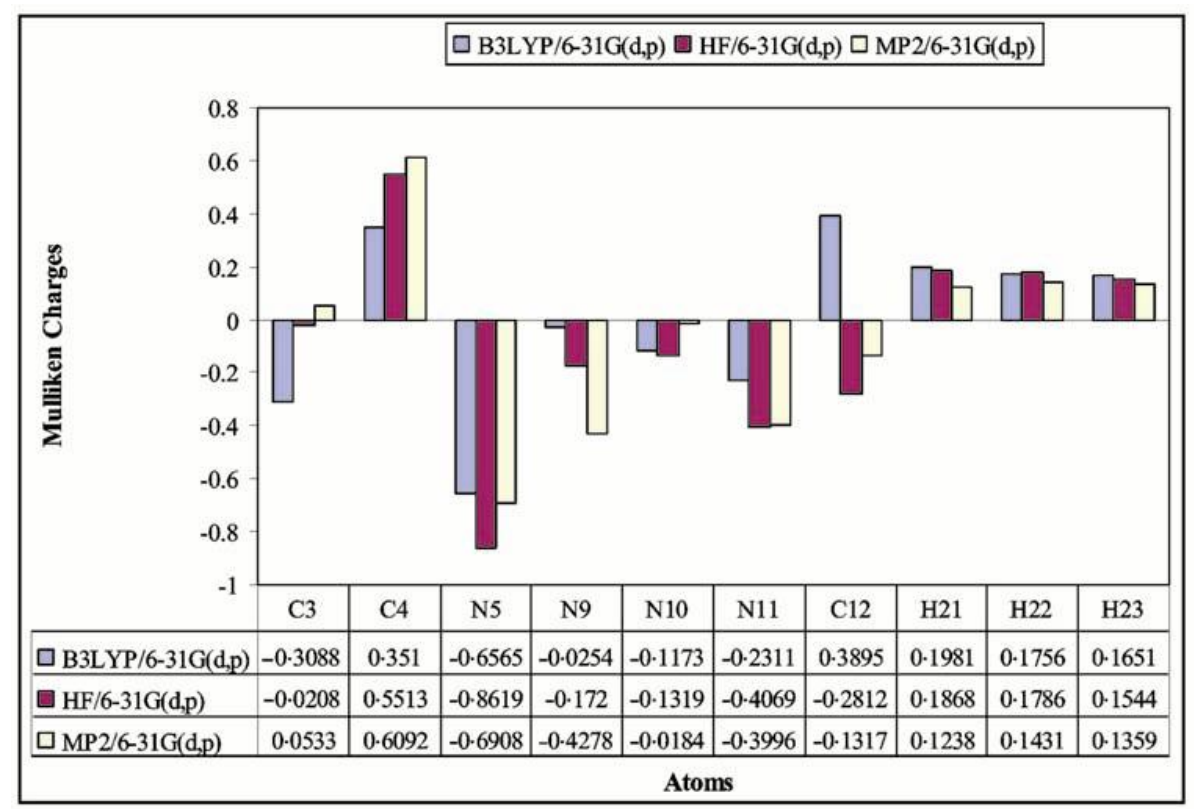

Figure 5. Comparison of different methods for calculated atomic charges.

$1496 \mathrm{~cm}^{-1}$. The $\mathrm{C}-\mathrm{NH}_{2}$ out-of-plane and in-planebending vibrations at 226 and $381 \mathrm{~cm}^{-1}$ observed at FT-Raman spectrum agree well with theoretically obtained values using B3LYP/6-31G $(d, p) .{ }^{19}$ 4.2c $C=N, C-N$ vibrations: The identification of $\mathrm{C}-\mathrm{N}$ vibrations is a difficult task, since the mixing of vibrations is possible in this region. However, with the help of the animation option of chemcraft 
Table 4. Calculated polarizabilities for dacarbazine.

\begin{tabular}{lcrrrrrr}
\hline Basis set & \multicolumn{1}{c}{$\alpha_{x x}$} & \multicolumn{1}{c}{$\alpha_{x y}$} & \multicolumn{1}{c}{$\alpha_{y y}$} & \multicolumn{1}{c}{$\alpha_{x z}$} & \multicolumn{1}{c}{$\alpha_{y z}$} & \multicolumn{1}{c}{$\alpha_{z z}$} & \multicolumn{1}{c}{$\langle\alpha\rangle$} \\
\hline B3LYP/6-311G & $204 \cdot 182$ & -10.604 & 118.330 & -0.002 & 0.003 & 55.637 & 126.049 \\
B3LYP/6-31G & 198.371 & -11.117 & 113.912 & 0.001 & 0.000 & $47 \cdot 120$ & 119.801 \\
B3LYP/6-31G* & 193.266 & 9.551 & 107.098 & 3.308 & 1.633 & 53.520 & 117.961 \\
HF/6-31G & 164.977 & 6.405 & $100 \cdot 136$ & -0.002 & -0.002 & 45.773 & 103.628 \\
MP2/6-31G & 106.556 & 5.545 & 73.206 & 2.706 & 1.950 & 23.133 & 67.363 \\
\hline
\end{tabular}

Table 5. Comparison of HOMO, LUMO, energy gaps ( $\left.\Delta \varepsilon_{\text {HOMO-LUMO }}\right)$, first ionization potentials and chemical hardness of dacarbazine $(\mathrm{eV})$.

\begin{tabular}{|c|c|c|c|c|c|}
\hline Basis set & $\varepsilon_{\mathrm{HOMO}}$ & $\varepsilon_{\mathrm{LUMO}}$ & $\begin{array}{l}\text { 1st ionization } \\
\text { potential }\end{array}$ & $\left(\Delta \varepsilon_{\mathrm{HOMO}-\mathrm{LUMO}}\right)$ & $\begin{array}{l}\text { Chemical } \\
\text { hardness }\end{array}$ \\
\hline B3LYP/6-311G & $-518 \cdot 706$ & $-1 \cdot 684$ & $-260 \cdot 195$ & $517 \cdot 021$ & $258 \cdot 510$ \\
\hline B3LYP/6-31G & $-519 \cdot 302$ & -1.477 & $-260 \cdot 382$ & $517 \cdot 825$ & 258.915 \\
\hline B3LYP/6-31G* & $-518 \cdot 952$ & $-1 \cdot 615$ & $-260 \cdot 283$ & $517 \cdot 337$ & $258 \cdot 668$ \\
\hline $\mathrm{HF} / 6-31 \mathrm{G}$ & $-557 \cdot 848$ & $2 \cdot 497$ & $-280 \cdot 172$ & $555 \cdot 351$ & 277.675 \\
\hline $\mathrm{MP} 2 / 6-31 \mathrm{G}$ & $-512 \cdot 650$ & $-1 \cdot 586$ & $-257 \cdot 118$ & $511 \cdot 064$ & $255 \cdot 532$ \\
\hline
\end{tabular}

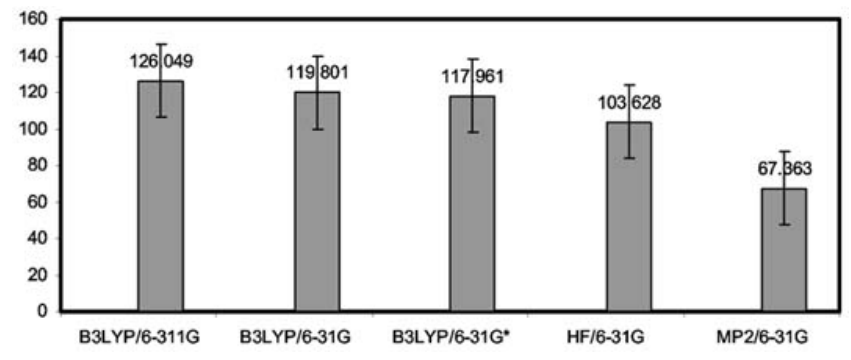

Figure 6. Comparison of different methods for calculated polarizabilities.

graphical interface for Gaussian programs the $\mathrm{C}-\mathrm{N}$ vibrations identified. Silverstein ${ }^{20}$ assigned $\mathrm{C}-\mathrm{N}$ stretching absorption in the region $1382-1266 \mathrm{~cm}^{-1}$ for aromatic amines. The IR bands appearing at 1510 and $1441 \mathrm{~cm}^{-1}$ are assigned to $v_{\mathrm{C}=\mathrm{N}}$ vibrations and 1255 and $1209 \mathrm{~cm}^{-1}$ are assigned to $v_{\mathrm{C}-\mathrm{N}}$ vibrations with the $\delta_{\mathrm{CH}}$ for the title compound. All these results agree with the results of Shanmugam et $a l^{21}$ and Sundaraganesan et al. ${ }^{22}$

4.2d Methyl group vibrations: The title compound dacarbazine, possesses two $\mathrm{CH}_{3}$ groups attached to the side chain of $\mathrm{N}=\mathrm{N}-\mathrm{N}$ to the five member ring. There are nine fundamentals one can expect to each $\mathrm{CH}_{3}$ group, namely the symmetrical stretching in $\mathrm{CH} 3\left(\mathrm{CH}_{3}\right.$ sym. stretch) and asymmetrical stretching (in plane hydrogen stretching mode); the symmetrical $\left(\mathrm{CH}_{3}\right.$ sym. deform $)$ and asymmetrical $\left(\mathrm{CH}_{3}\right.$ asym. deform) deformation modes; the in-plane rocking, out-of-plane rocking, twisting and bending modes. For the methyl compounds, the stretching mode appears in the range of $2825-2870 \mathrm{~cm}^{-1}$, lower in magnitude compared to its value in $\mathrm{CH}_{3}$ compounds $\left(2860-2935 \mathrm{~cm}^{-1}\right)$, whereas the two asymmetric modes for both the types of compounds lie in the same region of $2925-2985 \mathrm{~cm}^{-1}$. The FTIR bands at 2869 and $2755 \mathrm{~cm}^{-1}$ and FT-Raman band at 2851 and $2739 \mathrm{~cm}^{-1}$ represent the asymmetric and symmetric $\mathrm{CH}_{3}$ stretching vibrations of the methyl group of dacarbazine.

\section{Other molecular properties}

\subsection{Mulliken charges}

The calculation of effective atomic charges plays an important role in the application of quantum mechanical calculations to molecular systems. Our interest here is in the comparison of different methods to describe the electron distribution in dacarbazine as broadly as possible, and assess the sensitivity of the calculated charges to changes in (i) the choice of the basis set; (ii) the choice of the quantum mechanical method. Mulliken charges, calculated by determining the electron population of each atom as defined in the basis functions. The Mulliken charges calculated different levels and at different basis sets listed in table 3 . The results can, however, better be represented in graphical form as has been given in figure 5. From these results, it will be possible to 
Table 6. Theoretically computed energies (a.u.) zero-point vibrational energies ( $\mathrm{kcal} \mathrm{mol}^{-1}$ ), rotational constants $(\mathrm{GHz})$, entropies $\left(\mathrm{cal} \mathrm{mol}^{-1} \mathrm{~K}^{-1}\right)$ and dipole moment $(D)$ for dacarbazine.

\begin{tabular}{|c|c|c|c|c|c|}
\hline \multirow[b]{2}{*}{ Parameters } & \multirow[b]{2}{*}{ AM1 } & \multirow{2}{*}{$\underset{6-31 \mathrm{GF}(d, p)}{\mathrm{HF}}$} & \multicolumn{2}{|c|}{ B3LYP } & \multirow{2}{*}{$\begin{array}{c}\mathrm{MP} 2 \\
6-31 \mathrm{G}(d, p)\end{array}$} \\
\hline & & & $6-31 \mathrm{G}(d, p)$ & $6-311 \mathrm{G}(d, p)$ & \\
\hline Total energy & & $-634 \cdot 00265$ & $-638 \cdot 07212$ & $-638 \cdot 11876$ & $-638 \cdot 04222$ \\
\hline Zero-point energy & $115 \cdot 1$ & $124 \cdot 0$ & $118 \cdot 3$ & $113 \cdot 8$ & $116 \cdot 7$ \\
\hline \multirow[t]{3}{*}{ Rotational constants } & & $1 \cdot 4787$ & $1 \cdot 5202$ & $1 \cdot 4550$ & $1 \cdot 6342$ \\
\hline & & 0.4718 & 0.5007 & 0.4623 & 0.5007 \\
\hline & & $0 \cdot 3592$ & $0 \cdot 3943$ & $0 \cdot 3524$ & $0 \cdot 3941$ \\
\hline \multicolumn{6}{|l|}{ Entropy } \\
\hline Total & $104 \cdot 119$ & $111 \cdot 227$ & $101 \cdot 527$ & $115 \cdot 254$ & $92 \cdot 527$ \\
\hline Translational & $41 \cdot 355$ & $41 \cdot 561$ & $41 \cdot 505$ & $41 \cdot 505$ & $41 \cdot 505$ \\
\hline Rotational & $31 \cdot 562$ & $31 \cdot 528$ & $31 \cdot 277$ & $31 \cdot 584$ & $31 \cdot 277$ \\
\hline Vibrational & $31 \cdot 207$ & $36 \cdot 279$ & $29 \cdot 745$ & $42 \cdot 166$ & $19 \cdot 745$ \\
\hline Dipole moment & - & $6 \cdot 455$ & $5 \cdot 962$ & 5.998 & $5 \cdot 965$ \\
\hline
\end{tabular}
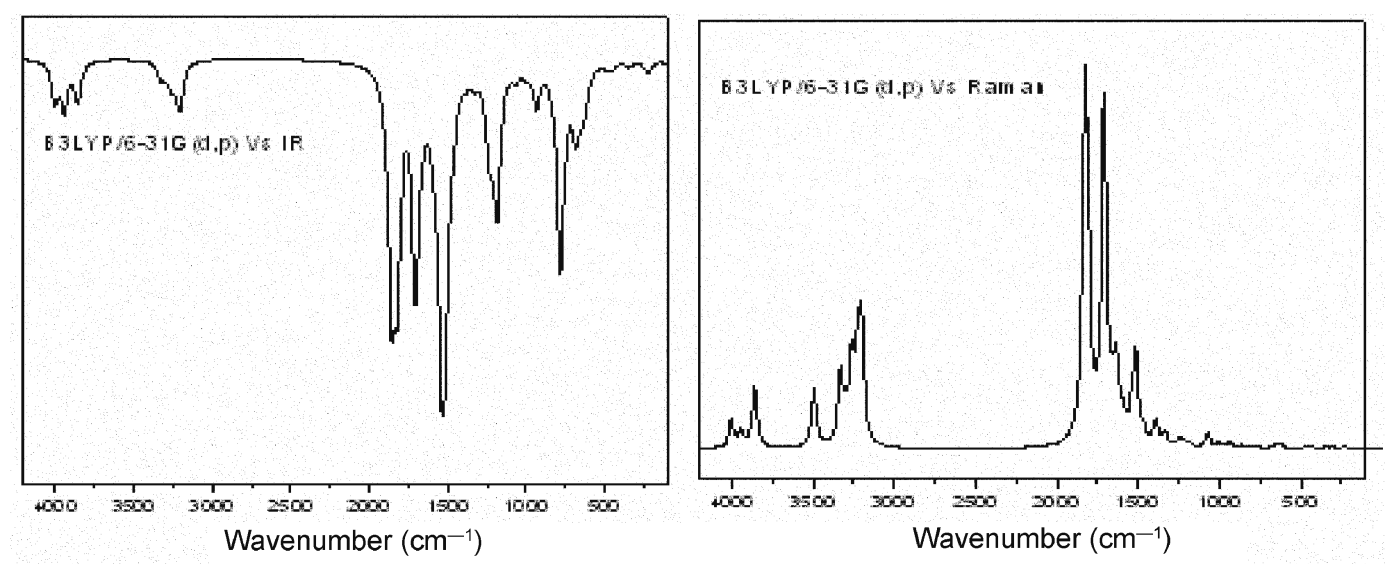

Figure 7. Computed spectra of dacarbazine at B3LYP/6-31G $(d, p)$.

say to the change to charge distribution by a change in basis set. The charges depending on basis set and are changed due to polarization.

\subsection{Molecular polarizability}

We have investigated the effect of the basis set on molecular polarizability of dacarbazine using Gaussian $03 \mathrm{~W}$. In this study, the computation of molecular polarizability of dacarbazine with different basis sets reported. Here, $\alpha$ is a second rank tensor property called the dipole polarizability and mean polarizability $\langle\alpha\rangle$ are evaluated using

$$
\langle\alpha\rangle=1 / 3\left(\alpha_{x x}+\alpha_{y y}+\alpha_{z z}\right) .
$$

The polarizability calculations carried out for different basis sets of dacarbazine are summarized in table 4. As seen from the figure 6 , the largest polarizability was observed for B3LYP/6-311G $(d, p)$.

\subsection{HOMO-LUMO energy gaps and ionization potentials}

According to Koopmans' theorem, $I=-E_{\mathrm{HOMO}}, A=-$ $E_{\mathrm{LUMO}}$. The ionization potentials and affinity potentials from Koopmans' theorem (or the HOMO and LUMO energies) for dacarbazine are summarized in table 5. The substitutional perturbation affects both the HOMO orbital and LUMO orbital for dacarbazine. In general, the HOMO orbital becomes less bound while the LUMO more bound with increasing heat of formation. From table 5, the lowest energy gap found at MP2 method.

\subsection{Thermodynamic properties}

The calculated thermodynamic parameters are presented in table 6. Scale factors have been recommended $^{23}$ for an accurate prediction in determining the zero-point vibrational energies and the entropy 
$\mathrm{S}$. The variation in the ZPVEs seems to be insignificant. The total energies found to decrease with increase of the basis sets. The changes in the total entropy of dacarbazine at room temperature at different basis sets are only marginal.

\section{Conclusion}

The results of the study lead to the following conclusions: (i) The frequency assignments performed for the first time from FTIR and FT-Raman spectra recorded were for dacarbazine. Theoretical DFT and $a b$ initio calculations of the vibrational spectra of the molecule presented in this paper were compared with the FTIR and Raman spectra. (ii) Geometries reported within the limits of accuracy of available experimental data. The molecular geometry of dacarbazine was best at the B3LYP level of DFT. (iii) Mulliken charges of dacarbazine at different levels were calculated and the results discussed. (iv) Molecular polarizability and ionization potential of dacarbazine were discussed and reported.

\section{References}

1. Vaughan K 1990 In The chemistry of antitumor agents (ed.) D E V Wilman (Glasgow: Blackie) pp 159-186

2. Carter S K and Friedman M A 1972 Eur. J. Cancer 85

3. Godin J R P, Vaughan K and Renton K W 1981 Can. J. Physiol. Pharmacol. 591234

4. Clarke D A, Barclay R K, Stock C C and Rondesvedt R S 1955 Proc. Soc. Exp. Biol. Med. 90484

5. Doucette K G, Pye Cory C, Vaughan Keith, Enright Thomas G 2006 J. Mol. Struct.: THEOCHEM 801 21-28
6. Carl Kemnitz 2002 Chemoffice ultra 10, Trial version

7. Young D C 2001 Computational chemistry: A practical guide for applying techniques to real world problems, John Wiley \& Sons Inc, p. 42

8. Chis V, Filip S, Miclaus V, Pirnau A, Tanaselia C, Almasan V and Vasilescu M $2005 \mathrm{~J}$. Mol. Struct. 744-747 363

9. Becke D 1993 J. Chem. Phys. 985648

10. Lee C, Yang W and Parr R G 1988 Phys. Rev. B37 785

11. Frisch M J et al 2004 Wallingford CT, Gaussian Inc

12. Grigoriy A Zhurko, Denis A Zhurko 2004 Chemcraft program, Academic version 1.5

13. Pulay P, Fogarasi G, Ponger G, Boggs J E and Vargha A 1983 Am. Chem. Soc. 1057037

14. Scott A P and Radom L 1996 J. Phys. Chem. US 100 16502

15. Satyanarayana D N 2004 Vibrational spectroscopyTheory and applications (New Delhi: New Age International (P) Limited Publishers) 2nd ed

16. Dollish F R, Fateley W G and Bentley F F 2001 Characteristics Raman frequencies of organic chemsitry (Orlando FL: Hartcourt College Publishers) 3rd edn

17. Bellamy L J 1980 The infrared spectra of complex molecules (London: Chapman and Hall) vol 2

18. Wiberg K B and Sharke A 1973 Spectrochim Acta A29 583

19. Sundaraganesan N, Meganathan C, Anand B, Dominic Joshua B and Lapouge C 2006 Spectrochim Acta 1386

20. Silverstein M, Clayton Basseler G and Morill C 1981 Spectrometric identification of organic compounds (New York: Wiley)

21. Shanmugam R and Sathyanarayan D 1984 Spectrochim Acta A40 764

22. Sundaraganesan N, Kumar K S, Meganathan C and Joshua B D 2006 Spectrochim Acta $\mathbf{A 6 5} 1186$

23. Alcolea Palafox M 2000 Int. J. Quantum. Chem. 77 661 